\title{
The Effect of Using an Exercise and Nutrition Secure Email Message on the Implementation of Health Promotion in a Large Health Care System
}

\author{
Travis Michael Combest (D) ${ }^{1}$, Allison Howell (D) 1 , Harjinder Kumar ${ }^{1}$, Samer Koutoubi ${ }^{2}$, \\ Karen Cieslewicz ${ }^{3}$, and Joetta Khan (iD 1 \\ ${ }^{1}$ Walter Reed National Military Medical Center \\ ${ }^{2}$ American Public University System \\ ${ }^{3}$ The Office of Karen Cieslewicz, MD
}

\begin{abstract}
Background and Program: Secure email messaging is used to provide timely communication between healthcare providers and patients with medical information and education. There have been increasing rates of overweight and obesity among military healthcare patients. To address this issue, we utilized a secure email messaging system that is primarily used for medication refills. This commentary demonstrates the extent to which the message was viewed and how much the message was liked. Preliminary Results: Of the 16,020 individuals emailed, 7,011 participants (43.8\%) opened the email, and 1,023 patients (14.6\% of those who opened it) completed the survey. Satisfaction rate for the health promotion message was $77.2 \%$. Participants reported an intent to eat more fruit and vegetables (49.6\%), decrease portion sizes (38.8\%), and increase physical activity $(51.1 \%)$, while $33.6 \%$ reported having no intent to change. Conclusion: The majority were satisfied with the health promotion message. This email method enables a single provider to have increased contact with patients. It is unknown if this message reached higher risk or harder to reach participants, a patient subset that could benefit most from this type of communication. This study is unique in that to our knowledge it is the first one to use secure email messaging in the military healthcare system for health promotion.
\end{abstract}

(C) 2019 and CC-BY 4.0 licensed by the authors.

Keywords: secure email messaging, exercise and nutrition message, preventive digital messaging, preventive health

\section{Introduction}

The availability and increased use of electronic health communications (email and social media) has greatly improved health care access among consumers and patients by providing secure online access to records, increasing direct messaging to healthcare providers and providing an opportunity to empower patients with accredited and high-quality health information. (Osterman, 2009, Thimbleby, 2013). The use of secure email messaging helps strengthen health communication and adheres to Health Insurance Portability Accountability Act (HIPAA) requirements. This has been incorporated into the Veterans Health Affairs (VHA) for medication refills, health care reminders, and communication with the clinicians averaging 2.2 personal messages per week overall, and 4.23 messages per week among those clinicians who have been using the system for more than one year (Byrne, et al., 2009). Use of this modality has the potential benefits of having increased patient engagement with their provider and ability for increased and specific communication between healthcare providers and patients. Weingart et al. analyzed a secure electronic messaging system with the MedCheck program, an application that sends a secure email message to patients 10 days after a new or updated prescription, and asks if it was filled or if there were any problems with the medication. Majority (79\%) of patients opened their MedCheck message. Patients who responded to the MedCheck message reported more adverse drug effects than the nonresponders who did not respond to the message, but did so at an office visit (Weingart, et al., 2008). The secure email could allow more time 
and a more comfortable environment for the patient to explain health concerns digitially, and could act as an effective extension for the actual medical encounter.

\section{Program}

Change Health (previously Relay Health) is a secure email service that military health care system participants and their allied health providers can utilize to send and receive messages about healthcare questions, labs and order medications. The features of the system are on par with other nationwide civilian healthcare systems (Carepoint, 2015 and Relay Health, 2015). Utilization of the secure email healthcare messaging is a priority of the military health care system, in an effort to maximize utilization of health-related resources. Exercise and nutrition messaging had never been implemented through this system. With a limited number of exercise and nutrition providers, this modality was considered as an additional mechanism to create additional contact with the patient population and potentially address overweight and obesity rates in the military healthcare population. The population included all users who had signed up in the secure email messaging system that were assigned to the local hospital system. The secure exercise and nutrition email message was sent to all users and if the user consented, they received a survey. The survey assessed: 1) level of satisfaction 2) intent to change nutrition or exercise habits; 3 ) intent to make appointments with health care providers for overweight and obesity management; 4) ownership of an activity tracker; 5) current physical activity level and; 5) desired frequency of health promotion messaging. Participants were emailed over a three-week period. The study was approved through the Institutional Review Board through American Public University and Walter Reed National Military Medical System.

\section{Preliminary Results}

The exercise and nutrition secure email message that was delivered provided the following message: "Eating more fruits and vegetables, decreasing portion sizes and walking or exercising 30 minutes a few times a week can greatly help with weight loss and increase your quality of life." The email also contained follow up information for appointments with a dietitian and exercise physiologists in the health care system. The health promotion message was adapted based on a study by Puhl et al. (2013), who found that the most favorable reactions for weight loss public health messages for overweight and obesity included multiple topics such as fruits and vegetables, physical activity and portion sizes (Puhl, et al., 2013).

Table 1.

Survey Response Rates

\begin{tabular}{lrr}
\hline & $\begin{array}{r}\text { Completed } \\
\text { Responses }\end{array}$ & $\begin{array}{r}\text { Response } \\
\text { Rate (\%) }\end{array}$ \\
\hline Opened email message (out of 16,020 emailed) & 7,011 & 43.8 \\
Opened Survey (out of 7,011 who opened email) & 1,023 & 14.6 \\
Opened Survey and Consented (out of 7,011 who opened email) & 985 & 14.0 \\
\hline
\end{tabular}

The response rate for this study was $14 \%$ which is similar to other online survey response rates (Dillman, et al., 2009). Overall results related to the receipt of the message and utilization of the message and survey are provided in Table 1. A large number of participants (43\%) opened the email message which is encouraging. This demonstrates that this email messaging can act as a provider extender, providing health promotion information to a large number of individuals from a single healthcare provider. Additionally, of those who opened the survey and consented, over half $(51.9 \%)$ indicated they preferred monthly health promotion emails over quarterly, biannual, or no emailed messages at all.

The majority of the participants $(77.2 \%)$ were satisfied with the exercise and nutrition secure email message and only $2.3 \%$ were dissatisfied, however $20.6 \%$ of participants indicated a neutral level of satisfaction. Reasons for dissatisfaction 
or neutral satisfaction were not collected in this study. Participant's responses regarding the intent to change behaviors over the next month and are presented in Figure 1. Participants showed the greatest intent to change behavior for exercise $(51 \%)$ and eat more fruits and vegetables (49\%) over the next month. Slightly over one third (33.6\%) of respondents indicated that they had no intention to change any of their healthrelated behaviors in the next month.

Figure 1.

Participant Responses on Intent to Change Behaviors over the Next Month

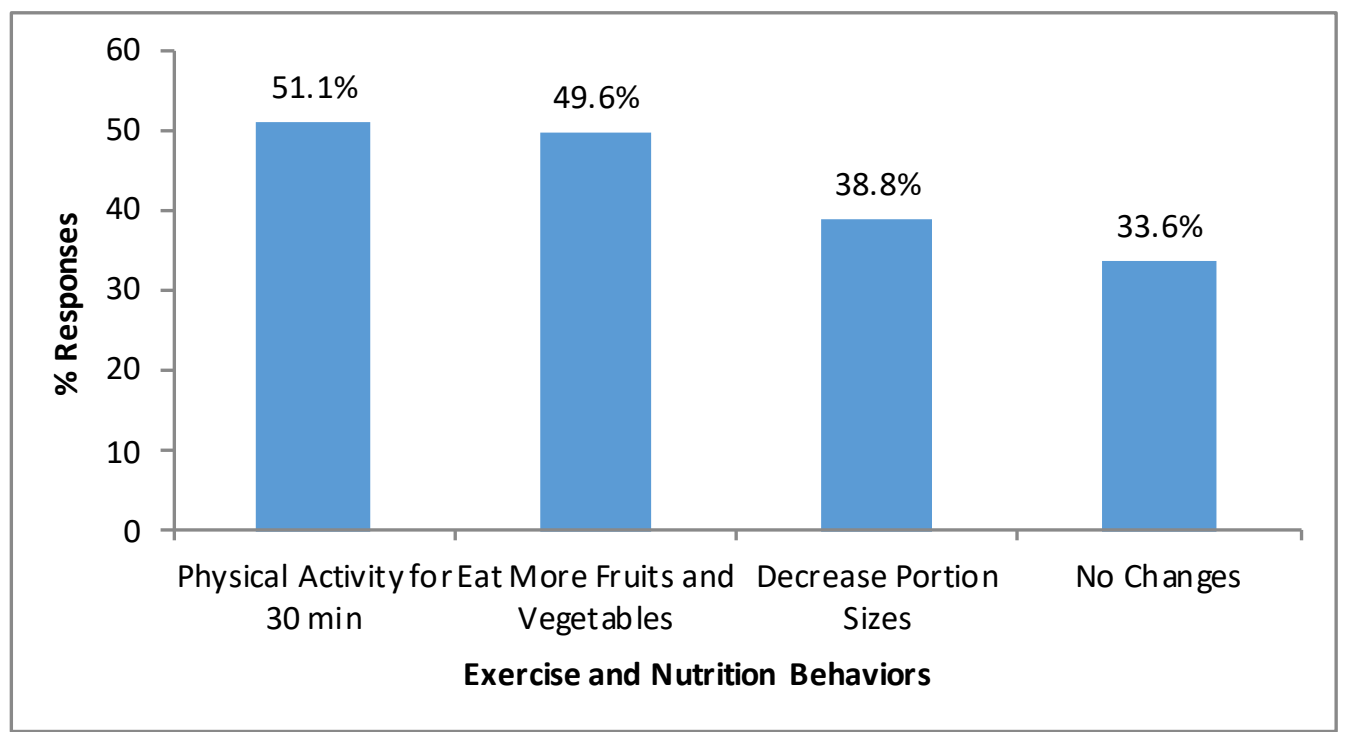

*Participants could select more than one exercise or nutrition behavior that they intended to change, $(n=973)$.

\section{Lessons Learned}

This study demonstrated that the use of the secure email messaging on nutrition and exercise messaging could be an effective method for health promotion. This modality could also be used to enable a healthcare system to come closer to reaching its entire population with a health promotion message. Further, the use of email messaging had a positive impact on self-reported intention to increase a variety of preventive health habits, which is important as health care resources become more constrained. However, despite the positive feedback and satisfaction for receiving health promotion messages through a secured email message, the study found that a remarkable number of subjects indicated no intention to make any change. Reasons for no intention to change where not collected, however, speculative reasons could include: participants already being fit or participants had heard similar health promotion messages before and therefore were not motivated to make changes. These findings could also indicate that more interventions are needed, individually tailored messaging, or the messages need to be more technologically advanced.

\section{Future Considerations}

Hypothesized future uses of health promotion messaging in the nutrition and exercise physiology fields could include contacting patients both before and after scheduled appointments. The health messaging sent before a nutrition appointment, for example, could be used to create anticipation or for dialogue during the appointment. Furthermore, an email message after the nutrition appointment could be used to maintain momentum towards reaching nutrition goals.

There is great potential for a future study for the usage of health promotion messaging on a large scale. For example, if there are 10,000 participants in a healthcare system and around $40 \%$ of this group are identified as being overweight/obese a single health promotion message could potentially reach thousands of 
individuals. Additionally, a certain percentage of those participants might be influenced by an emailed health promotion message to either: 1) schedule appointments for assistance with weight management as needed or 2) make some lifestyle changes for weight loss on their own. Health promotion messaging can be an extension of the healthcare that is provided and can further support patient health goals. This type of study could also have clinical relevance in identifying characteristics of individuals who are influenced by emailed health promotion messages versus those who are not, allowing clinicians to tailor who they enroll to receive messaging versus follow-up in person.

To assess the effectiveness of emailed health promotion messaging, future studies should include a prospective randomized controlled study design that assesses the influence of health promotion email messaging on body weight, waist circumference, blood pressure, and other lifestyle and health-related measures. Future studies should consider targeting at-risk populations, including those who are clinically overweight or obese. Future studies should also assess participants' readiness to change their lifestyle behaviors, so that messaging is tailored based on stage of readiness to change. The tracking of healthcare appointments booked following the receipt of email messages would be one indicator of success. Future studies should also consider assessing if type of provider changes the influence of the health promotion messaging (e.g. patient's physician vs. a dietitian or exercise physiologist).

Despite the great potential benefits of health messaging, potential barriers should be addressed. Specifically, some patients may have limited understanding of technology or a relative lack of health literacy. Additionally, increased email communications may cause this health promotion message to be considered among "junk" or "spam" email and automatically filtered. Simple instructions given by providers and administrative staff could help extend its usage and alleviate these barriers.

\section{Conclusions}

Within this military healthcare system, secure email messaging was an effective mechanism for a single provider to efficiently reach a large group of participants with a positive effect on participants' intent to eat more fruit and vegetables, and walk or exercise 30 minutes a few days a week. Having regular communication with health promotion messaging and follow up appointments with health care providers could potentially help in decreasing overweight and obesity rates in the military healthcare population. More work is needed to examine if the health messaging which measured intent actually lead to long term changes in health behavior, evidenced by appointments to healthcare providers or such outcome markers, such decreased weight and cholesterol, that can be tracked in the healthcare system.

\section{Disclaimer}

The identification of specific products or scientific instrumentation are considered an integral part of the scientific endeavor and does not constitute endorsement or implied endorsement on the part of the authors, Department of Defense (DoD), or any component agency. The views expressed in this manuscript are those of the authors and do not reflect the official policy of the Department of Army/Navy/Air Force, Department of Defense, or U.S. Government.

\section{Acknowledgements}

We would like to acknowledge Dr. Asma Bukhari, PhD, RD, Chief Nutrition Services, Walter Reed National Military Medical Center at Bethesda for editing, revisions and manuscript submission assistance. We would also like to acknowledge Dr. Lyubev Tmanova, Informationist and Biomedical Librarian, Walter Reed National Military Medical Center at Bethesda for editing, revisions and manuscript submission assistance. 


\section{References}

Byrne, J., Elliott, S. \& Firek, A. (2009). Initial Experience with Patient-Clinician Secure Messaging at a VA Medical Center. Journal of the American Medical Informatics Association, 16(2), 267-270.

CarePoint. (2015). Clinical processing software, enrollment data. Accessed on 12 AUG 2015.

Dillman, P., Phelps, G. \& Tortora, R.,et al. (2009). Response Rate and Measurement Differences in Mixed Mode Surveys Using Mail, Telephone, Interactive Voice Response and the Internet. Social Science Research, 38(1), 1-18.

Gill, D., Jones, G., Zou, G. \& Speechley, M. (2012). Using a single question to assess physical activity in older adults: a reliability and validity study. BMC Medical Research Methodology, 12, 20-27.

GraphPad. (2015). Quick Stats Wald Method Confidence Intervals. GraphPad Website http://www.graphpad.com/quickcalcs/confInterval2/. Accessed on 10 September 102015.

Kim, JY, Lee KH \& Kim SH, et al. (2013). Needs analysis and development of a tailored mobile message program linked with electronic health records for weight reduction. International Journal of Medical Informatics, 82(11), 1123-1132.

Liederman, E., Lee, J., Baquero, V. \& Seites, P. (2005). Patient-Physician Web Messaging, The Impact of on Message Volume and Satisfaction. J. Gen. Intern. Med, 20: 52-57. Miller, L. and Aharoni, E. Understanding Low Survey Response Rates Among Young U.S. Military Personnel. RAND Research Report. 2015; 1-53.

Osterman Research, Inc. (2009).The Importance of Social Networking Tools Relative to Conventional Tools. Osterman Research, Inc. Web site. https://www.ostermanresearch.com/home/surveyreports/. Published May 2009. Accessed on 11 AUG 2015.

Puhl, R., Peterson, JL \& Luedicke, J. (2013). Fighting obesity or obese persons? Public perceptions of obesity-related health messages. Intern. Journal of obesity. 37: 774-782.

Relay Health. (2015). Clinical Solutions. Relay Health Website. http://www.relayhealth.com/solutions/clinical-solutions. Published 2015.Accessed on August 12, 2015.

Thimbley, H. (2013). Technology and the future of healthcare. Journal of Public Health Research. 2:160169.

Vassar Stats. (2015). Confidence Interval of a Proportion. Newcombe and Wilson. Vassar Stats Website. http://vassarstats.net/prop1.html. Published 2015.Accessed on September 15, 2015.

Weingart, S., Hamrick, H., Tutkus, S. \& Carbo, A. et al. (2008). Medication Safety Messages for Patients via the Web Portal: The MedChek Intervention. International Journal of Medical Informatics, 77, 161-168.

Corresponding Author Information

Travis Michael Combest (ORCID: 0000-0003-4288-6762)

Walter Reed National Military Medical Center

8901 Wisconsin Avenue

Bethesda, MD 20889-5600

Telephone: (301) 400-2703,

email: travis.m.combest.civ@mail.mil 